\title{
BLIND SPOTS, THE REAR VIEW MIRROR, AND A ROAD MAP FOR CULTURAL DIVERSITY
}

\author{
Cindy Lindsay \\ Indiana University \\ Cathy Enz \\ Indiana University
}

Imagine a man driving down a street that he has driven everyday for the past 10 years. In the years that he has driven on this street, it has always been one-way. Over time, this driver has become so familiar with this street that he no longer looks out the windshield at the road, but drives while looking in the rear view mirror. At first, looking in the rear view mirror was just an interesting way of driving for this man. More recently however, since he had never been in an accident while driving by looking in the rear view mirror, he has come to believe that this is a safe and effective manner of driving. Now imagine that, unbeknownst to this man, the street has become, for the first time today, a two-way street. Since the man does not know of the change, he is driving on the road as if it is still one-way and he continues to look exclusively in the rear view mirror to navigate. He sees in his rear view mirror cars swerving as they pass. He can see the drivers of these other cars swearing at him. He wonders what has gotten into these people; why are they going the wrong way? Why are they acting so angry with him? He has not done anything wrong. He has been a model driver for 10 years. What is wrong with these crazy people? He thinks they are going to get him into an accident. He begins to panic. He grips the steering wheel and desperately scans the scene in the rear view mirror, trying to avoid an accident. As he scans the road he notices that some other cars (though not the regulars on this route) are going the same way as always. This, he thinks, proves that the drivers going the other way are wrong.

Meanwhile, the drivers on the new two-way road wonder what is wrong with this man. Can't he see that things have changed? They try desperately to warn him. They honk and yell, but he just looks at them as if they are crazy.

The man in this story is not unlike the author of "The Problems of a Man Teaching Some Women About Women" He has found himself, without warning, on a two-way street that he believes is one-way. He is 
confused and angered by those who appear to be going the wrong way, and he wonders why they are angry with him. Still he tenaciously clings to driving through traffic by looking exclusively in the rear view mirrorby navigating the future by looking to the past. If he would only change his perspective-drive by looking out the windshield-much of his confusion would be resolved. We will suggest in this article that Fernando Bartolome do just that-change his perspective to look at the street ahead-the future.

\section{What the Two-Way Street Ahead Looks Like}

The world of business and business schools is changing rapidly. No longer are organizations staffed primarily or exclusively by white males. With the increasing numbers of women and minorities in the work force, businesses are becoming two-way streets. Organizations are increasingly multiracial and multicultural in nature. In these diverse organizational environments, new forms of work and social interaction are needed. Looking at the past to extrapolate the needs of the future is no longer an effective means of determining interaction patterns for this new diverse work force. Teaching everyone to act and think like a white male or to act in stereotypical patterns of deference to white males are not effective solutions to navigating the complexity of these diverse conditions.

The good news is that dealing with this new two-way street is manageable. By: (1) facing the road ahead and driving while looking out the windshield, (2) knowing the rules and feel of the road, (3) being courteous to other drivers, (4) looking out for construction, and (5) understanding the benefits of a two-way street, most accidents can be avoided and maximum traffic flow facilitated. Herein lies the hope for Fernando and those like him.

\section{Strategies for Managing the Two-Way Street}

\section{Facing the Road Ahead and Looking out the Windshield-an Issue of Awareness and Responsibility}

How does one face the road ahead? The first step is to figure out who you are and where you are on the road-to look around you and to look at yourself. In the case of Fernando, he appears to still be looking in 
the rear view mirror. He sees only the outcomes of what he assumes to be others' faulty behaviors. As a result, Fernando does not take responsibility for the dynamics he describes occurring in his classroom. All of the explanations he offers for the negative reactions of his students are attributed to the styles and faults of the women students. By contrast, Fernando makes multiple references to his own superior ability as a teacher and his positive past performance. Though the data is insufficient to make a clear determination, it may well be that Fernando has not yet taken responsibility for his part in the dynamics he describes; he has not looked around himself, nor at himself. Thus, the first suggestion to Fernando is to be aware of himself and his place on the road. To do this he must take responsibility and share the blame for current negative dynamics and for past inequities.

Facing the Road. Facing the road ahead involves a shift in perception from the rear view to the front view. Rear view perception defines present data in terms of past accomplishments and trends. Front view perception accepts present data in whatever form they are presented and looks for future trends. Fernando appears to define his teaching ability, and thus his response to the present problems in class, in terms of past accomplishments. He therefore concludes that he cannot be at fault, and others must be wrong. Facing the road ahead means learning to deal with what comes rather than with what has been. To do this, it becomes important to know the rules and the feel of the road ahead.

\section{Knowing the Rules and Feel of the Road}

Knowing the Rules. In the case of Fernando, the new rules of the road are contained in the data based information on women in organizations. Simply reading popular works and anecdotal accounts (novels and magazines) is not enough. The kind of nuts and bolts road manual provided by data based research is needed. Some may feel that this is asking too much. After all, it is difficult enough to keep up with the research in one's own interest areas much less add the areas of minorities and women in organizations. However, a base of general knowledge of research regarding minorities and women in organizational settings can be acquired without a great deal of reading. For example, Gary Powell's book, Women and Men in Organizations, which could be read easily and quickly, is the kind of road manual that would provide a wide foundation of research on women in organizations. In addition to developing a general base of knowledge, specific studies relating to class topics and teacher interest areas could be explored in more depth. 
Ideally, the teacher would become not only knowledgeable about diversity, but also a resource for students dealing with their own experiences on the road. Fernando may not possess this type of general or specific knowledge. He says that he has read about "women's experience" (possibly indicating having read popular or anecodotal material) and that (when asked) he presents his "beliefs," but the women in the class cite research to prove him wrong. The best defense in this case is a good offense. Check sources and keep informed so that the rules of the road are known.

Getting the Feel. Reading the road manual and maps, however, does not tell you what the real experience of the road will be like. By just looking at the rules or maps, the driver is unaware of the changes in road conditions and misses the zest of the experience. Getting a feel for the road helps the driver deal with potential obstructions such as potholes, obscured corners, and unexpected construction detours. In addition, getting the feel of the road provides the driver with the added benefit of experiencing the hum of the motor and the view of the scenery. In the diverse classroom, getting the feel of the road can be as simple as taking seriously the feedback given by minority class members and incorporating the minorities' suggestions into future teaching behaviors. Such feedback would aid Fernando in determining the new nature of the two-way street. By taking in the information that the women in his class are offering, he may begin to experience the road he is on in his classroom. Other strategies may include team teaching with a minority faculty member or talking with minority faculty and friends about the issues and about their responses. More advanced strategies may include: (1) having students work in diverse groups for class exercises and class projects; (2) recognizing and discussing racist, sexist, ageist, and so forth language, behavior and assumptions as they arise in class and in one's self; and (3) providing cases, exercises, and examples which offer opportunity for reversal of traditional roles of minority and majority positions.

\section{Driver Courtesy}

All drivers are not created equally. Some may drive fast, others slow. Factors such as speed, experience, or style of driving may surface regardless of whether the road is one-way or two-way. In an ideal world all drivers would be created equally; but since they are not, it is the role of the responsible driver to at least treat all drivers with respect and courtesy. A willingness to look for and appreciate the strengths of all drivers should be a goal for all persons on the road. Courteous behavior 
to all drivers makes the experience of driving more pleasant. Clearly, in the classroom, students will differ in skills and abilities. However, a simple objective worthy of effort is to treat all participants with respect.

Respect for individual differences (beyond those due to racial or cultural differences) creates an atmosphere of principled learning. Caring for students and their ideas and opinions instills a sense of responsibility and self-respect. The teacher in a classroom, like the courteous driver, serves as a model for accepted behavior. Modeling the common courtesies of respect and caring can lead to the uncommon experience of a supportive and fun learning environment.

\section{Look Out for Constructions of Reality}

In addition to being aware, taking responsibility, getting information, and modeling courtesy, the driver must understand some aspects of the power and ethics of constructing realities. Let us imagine for a minute that the new two-way rules are in effect on the street but that all of the drivers on the street are looking in the rear view mirror, acting as if nothing has changed. Though the rules say the street should be two-way, it is, in effect, still one-way. Now imagine that one driver believes that the street is two way and ventures into traffic. This one driver is likely to be forced to turn around and treat the street as oneway or suffer the possible consequence of a head-on collision. Though the rules of the street say it is two-way, the traffic demands one-way actions. At this point our imaginations can allow us to see several possible future events. First, two-way drivers, as they venture one by one onto the new street, begin to learn that regardless of what they were told, this street is one-way. This is similar to the experience of women and minorities entering organizations in the 1960s, 1970s and even today. Second, if two-way drivers come in numbers to the street, there will be accidents, or at least a traffic jam. This is similar to the experience that Fernando is having in his class, an experience that many organizations are having now that the number of women and minorities in the work force has increased.

Let's take the analogy one step further and imagine that a police officer is on the street directing traffic. The legitimate right of the officer to direct traffic places the interpretation of the direction in which traffic should flow in his or her hands. More important, this interpretation is assumed to be the true reading of how traffic should flow. Those who do not comply with the officer's interpretation not only risk getting in an accident with other drivers, but also risk receiving a ticket. Everyone on the road is now dependent on the officer's skill in deciding and managing 
the flow and direction of traffic.

In the classroom, the teacher is the police officer, possessing legitimate power over students. To act like a responsible police officer, the teacher must be prudent, cautious, consistent, and cognizant of the well being of all students before directing and leading the class. Since the teacher's construction of reality is perceived by students to be legitimate, the ability to present differing constructions of reality may be advisable to the effective teaching of diverse students. A few ways in which alternate realities could be melded into the classroom might include: (1) inviting racially and culturally diverse guest speakers who are legitimate authorities (substitute cops) but provide alternative perspectives; and (2) selecting class leaders (student cops) to serve as the officers in charge of directing traffic, perhaps with the goal of leading the class down a new road or up a different alley. Finally, the teacher/cop may wish to create situations that are beyond the regular rules. The teacher may make the street one-way heading south some days, one way heading north other days, and two-way at other times. In essence, the teacher may wish to change the rules of the road to alter and try on different realities. Since the teacher's construction of reality is perceived as legitimate, the ethics of constructing realities must also be of concern to the effective teacher in a diverse classroom.

Thus, a primary ethical issue to be considered is whether or not one is already enforcing, or should attempt to enforce, a singular construction of reality in the classroom. To deal with this question we must first realize that even if one could get all others to view the road from the same perspective, not everyone would see the road the same way. Diversity of backgrounds and cultures results in differing perceptions from the same point of view. Even when promoting a particular construction of reality, valuing diversity entails allowing different visions from within that reality and understanding the benefit that can be derived from each perspective. Though we may all be looking out the front windshield, I may see the potholes to avoid, you may see the signs for the much needed upcoming pit stop, and someone else may be able to anticipate correctly the moves of the driver in front. All of these are valuable for effectively completing the journey. The teacher may choose to promote a particular construction of reality. However, valuing diversity requires that the teacher understand and utilize the perspectives that others bring with them to the classroom.

\section{Understanding the Benefits of the Two-Way Street-Valuing Diversity}

There are benefits to a two-way street. As we begin to value the 
benefits of diversity, the road becomes a more pleasant and prosperous place to drive. First, having people from diverse backgrounds and soliciting and valuing their input helps organizations to deal with the diverse and changing nature of market environments. Second, understanding and valuing diversity aids organizational members in working with others in the increasingly diverse work force. Third, cultural variety infused into the organizations by minority members can be effective toward achievement of organizational goals. ${ }^{1}$ To approach issues of diversity with these benefits in mind is to begin a journey of discovery-discovering optimal ways to promote and empower diverse peoples for organizational and societal advancement.

\section{Blind Spots, the Rear View Mirror and Managing Diversity}

The good teacher, like the good driver, can manage the diversity of the two-way street effectively if he or she will follow a few basic steps:

Face the road ahead:

1. Be aware of traffic. The road is no longer a one-way street.

2. Take responsibility. Know your role in the dynamics and take responsibility for your actions.

Know the rules and feel of the road:

3. Get data based information about diverse peoples and their experiences.

4. Absorb and learn from the experiences of minorities and women.

Exhibit driver courtesy:

5. Allow for variation in skills and abilities.

6. Model respect and caring.

Look out for construction of reality:

7. Teachers as traffic cops have the power to construct realities.

8. Permit substitute cops and student cops to provide multiple pictures of reality.

Understand the benefits of a two-way street:

9. Value diversity. 
In addition to suggesting these concrete steps, we believe it is important to note that driving while looking in the rear view mirror creates a specific blind spot-the future. People with past vision view life through the rear view mirror. The blind spot created by this is the tendency to base current and future actions and prescriptions solely on past events. When faced with new aspects of increased diversity in the classroom or corporation, the rear view mirror driver attempts to assimilate all participants into the norms and behaviors of the previous elite. Because of limited vision, the driver believes the street is still oneway and interprets others' actions on the basis of that belief. Thus, the driver applies old prescriptions and standards for behavior to the new people that enter the scene. In the business world, this leads to attempts to either train all people to think and act like white males or to act in stereotypical ways toward the existing white male hierarchy. The problem that arises with this approach is that it denies that the street has become two-way and in so doing, it diminishes positive effects of the new diversity that a two-way street provides.

Effectively managing cultural and ethnic diversity means understanding the differences between assimilation and integration in the acculturation of minorities in organizations and society (Berry, Wientrob, Sindell \& Mawhinney, 1982). Berry and associates suggest that in assimilation processes, minorities relinquish cultural identity and are absorbed into the dominant culture. This is similar to trying to convince the new drivers on the two-way street, that the street should be navigated as if it were still a one-way street. By contrast, the process of integration allows minority groups to maintain cultural integrity as well as become an integral part of the dominant culture. Integration processes accept the two-way street and attempt to maximize traffic flow given this diversity. Processes and attitudes of assimilation may eventually create equity but they diminish diversity; they create a false return to the one-way street. Successful processes of integration create equity while maintaining diversity-the new two-way street.

Fernando appears to take the assimilation (one-way street) approach. He describes in detail the types of women he likes and those he does not like. These descriptions lead us to conclude that Fernando values women who fit in his dominant culture but does not value those who represent diverse attitudes and styles. This kind of attitude toward minorities creates expectations that the minority member must change to fit the previously dominant culture, and it eventually results in the 
loss of possible attributes of diversity-

We believe that the use of an assimilation approach is based in the blind spot created by looking in the rear view mirror. The assimilation approach assumes that forming the future in terms of the past is optimal. Training diverse peoples to act like or in accordance with the unitary culture of the past does not truly aid these diverse people. It is our hope that all teachers will serve as model drivers by looking straight ahead and not by looking in the rear view mirror. By striving to integrate, not assimilate, culturally diverse groups, we, as educators, will also learn and grow. Facing forward and dealing with cultural diversity is a challenge, but it is our belief that all drivers will benefit by seeing and experiencing the twists and turns of the roads ahead.

\section{Note}

For example, see the paper presented by Karen Korabik at the 1988 National Academy of Management Meetings, "Is the Ideal Manager Masculine?: The Contributions of Femininity to Managerial Effectiveness."

\section{Reference}

Berry, J., Wientrob, R., Sindell, P., \& Mawhinney, T. (1982). Psychological adaptation to cultural change among the James Bay Cree. Le Naturaliste Canadien, 109, 965-975. 